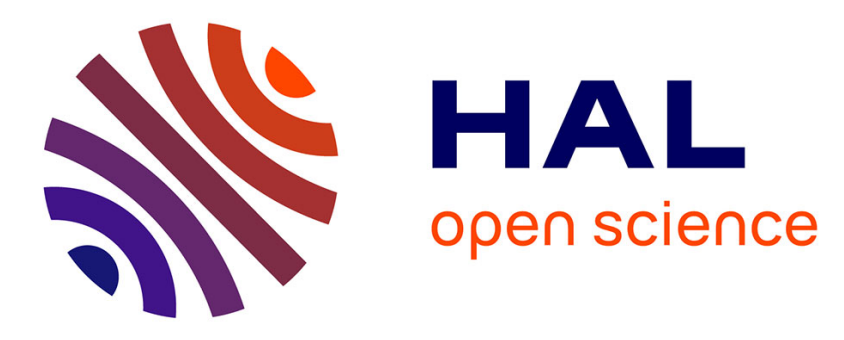

\title{
Entre idéal dépassé et norme imposée : une représentation figée du père projetée dans une société en mutation
}

\author{
Assadek Aziz, Adèle Assous, Bernard Golse, Magali Chabin-Fenlon, Corinne \\ Fernet-Lucas
}

\section{To cite this version:}

Assadek Aziz, Adèle Assous, Bernard Golse, Magali Chabin-Fenlon, Corinne Fernet-Lucas. Entre idéal dépassé et norme imposée : une représentation figée du père projetée dans une société en mutation: Quelle représentation de la fonction paternelle dans une institution sociale exerçant des mesures d'AEMO ?. Topique - Revue freudienne, 2016, Bébés et parents en difficulté, 2 (135), pp.159 - 173. 10.3917/top.135.0159 . hal-01508658

\section{HAL Id: hal-01508658 \\ https://hal.science/hal-01508658}

Submitted on 5 Jul 2017

HAL is a multi-disciplinary open access archive for the deposit and dissemination of scientific research documents, whether they are published or not. The documents may come from teaching and research institutions in France or abroad, or from public or private research centers.
L'archive ouverte pluridisciplinaire HAL, est destinée au dépôt et à la diffusion de documents scientifiques de niveau recherche, publiés ou non, émanant des établissements d'enseignement et de recherche français ou étrangers, des laboratoires publics ou privés. 


\title{
Entre idéal dépassé et norme imposée : une représentation figée du père projetée dans une société en mutation
}

\author{
Quelle représentation de la fonction paternelle dans \\ une institution sociale exerçant des mesures d'AEMO?
}

\author{
Aziz Essadek, Adèle Assous, Bernard Golse, \\ Magali Chabin-Fenlon, Corinne Fernet-Lucas
}

\section{QU'EST-CE QUI FAIT LE PèRE ?}

Évolution juridique et sociale du rôle du père

Le Pater Familias disparaît dans la législation. Ne survit-il pas pour autant dans les mentalités, et les institutions ne continuent-elles pas de le tuer? Nous interrogerons les effets du passage d'une autorité strictement paternelle à parentale.

La place des pères dans les institutions sociales et médico-sociales a toujours été source de tumulte. Cette place est intrinsèquement liée à l'évolution de leur position dans la société. L'autorité exclusivement détenue par le Pater Familias va être partagée avec les mères et l'État. À ce titre, Delion (2011), Lebrun (2011) ou encore Ferrand (2005) s'emploient à une historisation des lois sur le rôle des pères au sein de la famille en préalable à leurs réflexions sur cette évolution. Ce rappel est nécessaire pour introduire notre propos car l'éviction de cette réalité ne permettrait pas de prendre en considération la situation contemporaine des pères et donc de les lier aux institutions.

Selon l'Église au xII ${ }^{\mathrm{e}}$ siècle, le mariage est institué en sacrement, c'est donc le mariage qui fait le père. Celui-ci sera le garant de l'éducation des enfants. À la Renaissance, donneur de sens, il a charge d'éduquer l'âme de ses enfants.

Avec la mort de Louis xVI, la Révolution marque la déchéance de l'identité paternelle car le roi se présentait comme le «père » de ses sujets, ce qui n'était pas sans effet sur la représentation collective de l'image du père. Depuis la période révolutionnaire, la place du père était régie par le Code civil napoléonien (1804)qui s'inspirait lui-même du modèle judéo-chrétien (Ferrand, 2005). 
Le Code civil rétablit le père dans ses droits, il est à noter l'intervention de l'État pour en limiter abus et carences. On soulignera un glissement de la notion de droits vers celle de devoirs du père. En $1889^{1}$ les pères «indignes » sont surveillés par les préfets. La mère reste sous tutelle, mineure juridiquement à l'instar des criminels et débiles mentaux. L'État contrôle la puissance paternelle et on assiste à la naissance d'un paternalisme d'État sous la troisième République. De plus, les lois de Jules Ferry (1882) instaurent l'école obligatoire. L'autorité du père se partage avec celle du maître.

$\mathrm{Au} \mathrm{XX}^{\mathrm{e}}$ siècle, la paternité moderne est induite par de nouveaux modes de vie (industrialisation, exode rural, les deux guerres et l'arrivée des femmes sur le marché du travail). À cela s'ajoutera le chômage. En conséquence, la mère devient progressivement le seul parent présent physiquement au foyer. Et l'État la soutient avec la création de nouveaux métiers : assistantes sociales, juges pour enfants et médecins spécialisés. En $1935^{2}$, le «droit de correction paternel»est substitué par le placement de l'enfant en maison d'éducation surveillée, en institution. Cette «aideéducative»est miseen place sans passer parles pères pour régler les problèmes familiaux. En conséquence, émerge une nouvelle image des pères, celle de lacarence. 1968, les événements marquent la révolte contre l'Autorité dont celle des pères et une nouvelle avancée déterminante du mouvement d'émancipation des femmes.

La loi de 1970, va permettre d'introduire la notion d'égalité entre les sexes au sein de notre société, sur la question de l'autorité parentale. Elle est liée à l'arrivée massive de femmes diplômées sur le marché de l'emploi, à des changements politiques impulsés par les mouvements féministes et par le nombre croissant de couples divorcés (Ferrand, 2004). L'autorité paternelle va se transformer en autorité parentale partageant les responsabilités et les fonctions d'une manière égalitaire autant que possible (Senécal et al., 2015). Cette notion d'autorité parentale engage une nouvelle réflexion. Elle modifie la place des sexes dans les obligations éducatives, ce que Lebrun questionne autour du changement lexical de paternel vers parentalité : «Parental entraîne donc, sans le dire, un estompement de la différence des sexes et parentalité entérine sa disparition tout en maintenant la différence générationnelle. »(Lebrun, 2011,p.11-12). Cet aspect est essentiel à bien des égards, d'une part parce qu'il va substituer le rôle des parents - père, mère - à celui d'éducateur ; " Le mot de couple se réduit alors à désigner deux intervenants dans une symétrie exempte de toute référence à un réel qui le ferait coexister ensemble » (Ibid., p.12) et d'autre part parce que cette notion de parentalité va également bouleverser la position et la fonction paternelle. Avec l'abandon de l'autorité paternelle, c'est la mort du

1. Loi du 24 juillet sur la protection des enfants maltraités ou moralement abandonnées. Source : Journal officiel du 25 juillet 1889.

2. Décret loi du 30 octobre 1935. Source: Journal officiel du 31 octobre 1935. 
Pater Familias et son remplacement par l'autorité parentale. La famille devient un lieu d'égalitééducative.

On assiste peu à peu à un ajustement de la législation (1967 la pilule, 1975 l'IVG) qui va influer sur les nouveaux mode de vie. La femme a alors un droit unilatéral à l'IVG sans le consentement nécessaire de son mari. Cette évolution médico-sociale et législative vient renverser ce que l'homme avait mis en place pour asseoir sa domination masculine, à savoir un processus permettant de compenser sa faille narcissique générée par le fait que : «pater semper incertus est, tandis que la mère est certissima »(Freud, 1909c, p. 159). Cette domination trouvant sa source dans son incapacité à garantir seul sa descendance : «Pour se reproduire à l'identique, l'homme est obligé de passer par le corps de la femme. Il ne peut le faire par lui-même. C'est cette incapacité qui assoit le destin de l'humanité féminine. » (Héritier, 2012, p. 23).

Aujourd'hui, les évolutions médicales (contraception, IVG, procréation médicalement assistée) vont à l'encontre de ce processus de domination et apportent une liberté aux femmes en matière de sexualité mais viennent également modifier les principes d'accès à la parentalité « car les hommes doivent nécessairement passer par la volonté d'une femme pour devenir père » (Ferrand, 2005, p. 75). Autrement dit, le pouvoir de la femme dans ce contexte d'une contraception médicalement auto-réglée est immense. Il lui revient la possibilité de nier le désir de l'homme de devenir père, selon son désir à elle. Le pouvoir se trouve alors renversé par rapport à la période antérieure à cette révolution médicale. Ce changement de position des femmes dans la société a impacté la fonction paternelle. Celle-ci offrait un pilier à la virilité qui se voit alors fragilisée par un remaniement juridique et sociétal du pouvoir du père. La figure paternelle et celle de l'homme se confondaient, la notion d'autorité structurant l'un comme l'autre. Ce n'est plus d'actualité. L'autorité doit être redéfinie dans le couple conjugal, entraînant une modification de la place du père comme de la mère.

Actuellement, l'intérêt et les droits de l'enfant priment tandis qu'antérieurement l'autorité revenait exclusivement au père. Ainsi que l'énonce Jacob Alby et Vives:

«Nous soutenons que chaque être parlant, dit parlêtre, peut être porteur des fonctions parentales que nous résumons ainsi : être porteur d'un intérêt particularisé pour l'enfant et de la castration liée au langage, ceci indifféremment du sexe et du genre du parent concerné. » (2015, p. 27). En d'autres termes, il existe une dissociation entre l'intérêt porté à l'enfant et le sexe du parent. La notion de castration est symbolique et inhérente au langage quel que soit le genre parental.

La recherche d'un équilibre est de rigueur dans les familles dont le fonctionnement tend à être harmonieux mais qu'en est-il de cette parentalité lorsque les parents ne s'entendent plus? (Lebrun, 2011). Que reste t-il de la place des 
pères lorsque le conflit s'intensifie et que l'enfant est en péril ? Cette question prend son sens au regard de l'institution dans laquelle nous avons mené notre étude, un service mettant en œuvre des mesures d'Assistance Éducative en Milieu Ouvert(AEMO).

Ce type de structure nous invite à définir la problématique en ces termes : quelle place pour le père lorsque l'environnement de l'enfant est désigné comme défaillant, lorsque les conditions d'éducation mettent l'enfant en danger?

À partir d'une étude populationnelle réalisée sur 796 mineurs au sein d'un service mettant en œuvre des mesures d'AEMO, nous analyserons des données statistiques concernant la présence des pères dans l'institution, la rencontre avec les professionnels, l'investissement dans la mesure éducative et leur place de père au sein de la famille. Ceci afin d'établir une base quantitative qui nous servira de support pour analyser la représentation des pères élevée au statut de mythe. Nous discuterons de l'impact de ce mythe sur leur effort pour investir la fonction parentale. Pour compléter notre approche quantitative, nos propos seront étayés par des vignettes cliniques.

\section{CONTEXTE INSTITUTIONNEL DE L’AEMO}

L'ONED ${ }^{3}$ estime qu'en France, 284000 enfants sont concernés par des mesures de protection de l'enfance ${ }^{4}$ et que les mesures éducatives en milieu ouvert concerneraient 154800 situations. Ces mesures ordonnées par un juge des enfants, suite à un signalement, sont souvent des alternatives aux placements permettant de poursuivre la prise en charge tout en mettant la famille au centre du travail. Ce dispositif de $1^{\prime}$ AEMO qui vit le jour en $1958^{5}$, réaffirmé par la loi de 2007, permet d'accompagner le mineur dans son environnement familial tout en limitant les risques qu'il encourt.

Dans son article sur l'institution et la place des familles en protection de l'enfance, Vicente et al., (2014) soulignent la complexité des liens familiaux dans les familles suivies par des services exerçant des mesures de protection de l'enfance où il est difficile pour l'enfant de devenir «je».

Ainsi le rôle des institutions et notamment du magistrat est « de mettre socialement un interdit et des limites à des situations où des adultes n’ont pu établir, dans leur propre fonctionnement psychique, les limites et les interdits qui se

3. Source : Dixième rapport au Gouvernement et au parlement de l'Observatoire Nationale de l'Enfance en Danger (ONED) Mai 2015. Selon le rapport, les chiffres sont une estimation au 31 décembre 2012.

4. Aide Éducative à domicile, Accueil provisoire, Placement provisoire, AEMO.

5. Ordonnance du 23 décembre 1958 relative à la Protection de l'Enfance et de l'Adolescence en danger. Art. 375 du code civil français. 
transmettent normalement de génération en génération. [...] Le recours à la loi est thérapeutique dans la mesure où il peut être l'occasion de rappeler qu'il y a, en droit, un ordre possible et repérable pour remédier au dysfonctionnement familial, quand les places et les fonctions de chacun ne sont ni définies, ni assignables » (Clément, 1986, p. 164, cit. par Vicente et al., 2014, p. 37). Dans le cadre de l'AEMO, l'éducateur spécialisé en charge de la mesure sera le représentant du juge des enfants et apportera une triangulation à la « dyade parent-enfant ». Cette intervention, support de la fonction parentale, soutient spécifiquement les enjeux de différenciation. Le professionnel intervienten mettant du tiers pour parer à une défaillance de la triangulation qui donne lieu à une relation symbiotique ou abandonnique mère-enfant. La pérennisation de ce type de relation a été soulignée à de nombreuses reprises comme pathogénique. Dans cet équilibre des places se joue un enjeu de dialogue que Golse énonce: «Pour pouvoir parler à (quelqu'un), encore faut-il être hors de, sans fusion, sans symbiose. » (Golse, 1990). L'accès à la symbolisation augure de la construction du roman familial. Or l'évolution des systèmes familiaux rend complexe la triangulation et la construction du roman familial.

Nous ne souhaitons pas résumer toutes les situations suivies par les services d'AEMO dans cette problématique ; toutefois il nous semble que nombre d'entre elles ont pour origine de leurs dysfonctionnements une dissymétrie de l'intersubjectivité parentale.

\section{OUTILS ET MÉTHODOLOGIE}

L'étude populationnelle a été réalisée, en concertation avec le département Management des Organisations du Secteur Associatif de l'Université Paris-Est Créteil, auprès des 796 enfants suivis par un service associatif habilité exerçant des mesures d'AEMO de la région Île de France. Un questionnaire comprenant 5 items a été distribué à tous les travailleurs sociaux afin qu'ils le renseignent pour chacun des mineurs accompagnés.

\section{La situation del'enfant}

Cet item permet de nous informer sur l'état civil (le questionnaire anonymisé (codes) comportait l'âge, le sexe de l'enfant et sa position dans sa fratrie), la scolarité ainsi que sur la situation familiale de l'enfant. Dans ce cadre là, nous répertorions qui détient l'autorité parentale, la mère, le père, le tuteur ou un Tiers Digne de Confiance (TDC). Sont également interrogés et évalués les contacts entre l'enfant et le père ou l'enfant et la mère. Nous interrogeons de même, l'existence du Droit de Visite et d'Hébergement (DVH) exercé par le père, la mère ou autre. On notera l'éventuelle mise en place d'une restriction 
ou d'une suspension des DVH. Enfin, on interroge le passé de l'enfant, à savoir s'il a été élevé par les parents conjointement, le père seul, la mère seule ou encore s'il a été pris en charge par d'autres dispositifs : sanitaire, administratif, judiciaire ou encore familial élargi...

2. Les facteurs socio-économiques actuels de la famille

Cet item nous permet de recueillir des informations sur les facteurs socioéconomiques actuels de la famille. Cinq grands axes sont développés. Le premier questionne le type d'habitat de l'enfant. Le deuxième précise avec qui l'enfant partage son lieu de vie habituel, si les parents vivent dans la même commune ou département. Le troisième axe interroge la capacité de l'enfant et de la famille à se déplacer jusqu'au service. Le quatrième porte sur la situation professionnelle des parents. Le cinquième axe enquête sur la situation conjugale des parents.

3. Les mesures judiciaires antérieures et actuelles

4. Le signalement

5. Le travail en partenariat

Dans le cadre de cet article, nous centrons notre intérêt essentiellement sur la situation de l'enfant et les facteurs socio-économiques actuels de la famille (items 1 et 2 de cette enquête).

Le système de cotation consiste à attribuer un point par réponse cochée. Les résultats sont ensuite convertis en pourcentage.

Ce questionnaire a été construit et validé par la direction du service d'AEMO et l'ensemble du personnel a pu, s'il le souhaitait, contribuer à la réflexion et à son élaboration.

\section{RÉSULTATS}

À noter en préalable que les données recueillies sont strictement relatives à une institution, en l'occurrence un service d'AEMO. Les résultats sont propres à cet établissement. Nous n'avons pas répertorié d'étude similaire qui permettrait une étudecomparative.

Sur l'ensemble des 1046 questionnaires distribués 796 nous ont été retournés complétés par les éducateurs. $53 \%$ concernent des garçons et $47 \%$ des filles. 
Tableau 1 : Résultats en pourcentages de l'étude populationnelle sur les items 1 et 2

\begin{tabular}{|c|c|c|c|c|}
\hline & Père seul & Mère seule & $\begin{array}{l}\text { Parents } \\
\text { (Père + } \\
\text { mère) }\end{array}$ & $\begin{array}{c}\text { - Tiers Digne } \\
\text { de Confiance } \\
\text { - Tuteur }\end{array}$ \\
\hline Détenteur(s) de l'autorité parentale & 3,34 & 13,74 & 79,82 & 3,09 (tuteur) \\
\hline Détenteur(s) de l'autorité parentale & 3,92 & 33,7 & 50,2 & $12,18(T D C)$ \\
\hline $\begin{array}{l}\text { Parents séparés ne vivant pas } \\
\text { dans la même commune }\end{array}$ & - & - & 58,5 & - \\
\hline $\begin{array}{l}\text { Parents séparés ne vivant pas } \\
\text { dans le même département }\end{array}$ & - & - & 38,5 & - \\
\hline Parents ayant une activité professionnelle & 61 & 45,8 & - & - \\
\hline Parents étant sans activité professionnelle & 39 & 54,2 & - & - \\
\hline \multicolumn{5}{|l|}{ Contact de l'enfant avec ses parents : } \\
\hline - $\quad$ Régulier & 39,4 & 71,87 & \multirow{6}{*}{-} & \multirow{6}{*}{ - } \\
\hline - Continu & 16,47 & 11,15 & & \\
\hline - Irrégulier & 15,67 & 6,73 & & \\
\hline - Interrompu & 20,72 & 9,02 & & \\
\hline - $\quad$ Parent inconnu & 7,7 & 1,07 & & \\
\hline - $\quad$ Non renseigné & 0,4 & 0,16 & & \\
\hline Droit de visite et d'hébergement : & & & & \\
\hline $\begin{array}{l}\text { (sur l'ensemble des DVH/sur l'ensemble } \\
\text { des mesures) }\end{array}$ & & & & \\
\hline - $\quad$ Exercé (359/796) & $54-24,4$ & $38,5-17,3$ & $7,5-3,4$ & \multirow{3}{*}{-} \\
\hline - $\quad$ Restriction (60/796) & $60-4,5$ & $40-3$ & - & \\
\hline - $\quad$ Suspension (59/796) & $52,5-3,9$ & $47,5-3,5$ & - & \\
\hline Enfants dont les parents sont séparés & - & - & 76 & - \\
\hline
\end{tabular}


À la lecture des résultats de cette étude populationnelle, une tendance générale se dessine. Nous pouvons souligner l'importance des situations familiales où les parents sont séparés $(76 \%)$. Ce qui est plus révélateur est la prédominance des mères, lorsque les parents sont séparés, à s'occuper de leurs enfants. Nous pouvons croiser ces données avec le rapport du ministère de la justice intitulé «La résidence des enfants de parents séparés, de la demande des parents à la décision du juge ${ }^{6}$ ». Il s'avère que sur l'échantillon de 6042 décisions définitives, la résidence chez la mère a été prononcée dans $71 \%$ des situations contre $12 \%$ chez le père, quand $17 \%$ concernent des résidences en alternance.

Notre étude souligne que lorsque l'un des deux parents élève seul son enfant, il s'agit majoritairement de la mère $(33,7 \%$ contre $3,92 \%$ pour le père). Nous retrouvons cette tendance dans l'attribution de l'autorité parentale. En effet, si celle-ci n'est pas conjointe, la mère se trouve également plus désignée par le juge des enfants $(13,74 \%$ contre $3,34 \%$ pour le père).

Concernant la régularité des rencontres entre les parents et l'enfant, il apparaît que les mères sont davantage présentes que les pères. Quand 83,02\% des mères maintiennent un contact régulier ou continu avec leurs enfants, seuls $55,87 \%$ des pères le font. L'ensemble de ces données souligne une plus grande présence au quotidien des mères.

Cette tendance est à mettre en lien avec un nombre plus important de mères sans activité professionnelle que de pères. Ce qui contribue, probablement, au fait qu'elles seront plus présentes dans les institutions. En d'autres termes, elles ont majoritairement la résidence de l'enfant, elles maintiennent plus le contact avec leurs enfants et sont donc plus enclines à les accompagner. Cependant, cette interprétation est à nuancer car l'écart $(15,2 \%)$ entre la proportion des mères travaillant $(45,8 \%)$ et celle des pères $(61 \%)$ n'est pas explicatif à lui seul. Cet écart dans notre population est très similaire à celui observé à l'échelle nationale $\left(14,9 \%^{7}\right)$. La difficulté des professionnels à rencontrer les pères dans les institutions ne peut-être expliquée à elle seule par l'activité professionnelle. L'éloignement géographique est également à prendre en considération. En effet, il est important de souligner que plus d'un père séparé sur deux $(58 \%)$ ne vit pas dans la même ville que la mère et plus d'un père sur trois $(38,5 \%)$ ne vit pas dans le même département.

6. Source: Rapport du ministère de la justice, publié en novembre 2013. L'étude porte sur 6042 décisions définitives de justice sur les 8264 relatives ou définitives qui ont été établies en France entre le 4 et le 15 juin 2012.

7. Source: Le taux d'emploi des hommes et des femmes, INSEE, 2011. 


\section{DISCUSSION}

Nous constatons, dans ce service, que les pères sont peu présents physiquement au quotidien. Leur activité professionnelle, l'éloignement géographique, l'attribution de la résidence sont à prendre en considération.

Ces éléments peuvent être source de controverse : éloignement signifie-t-il nécessairement abandon ? Désertification des responsabilités ? Est-ce en lien avec la stigmatisation judiciaire induite par la mesure d'AEM ? La distance estelle toujours de la responsabilité du père ? Que les pères soient loin veut-il pas dire nécessairement qu'ils se soient éloignés d'eux-mêmes ? Ne peuvent-ils pas, dans certaines situations, avoir été mis à distance en partie par les mères et/ou les institutions elles-mêmes?

L'histoire même de la construction des institutions sociales peut apporter des éléments explicatifs. Rappelons que l'aide sociale a eu pour vocation première de protéger l'enfant et d'aider les mères dans des situations où le père était défaillant, violent ou absent. De fait, les institutions se sont créées, avant tout, autour de l'enfant. La place de la mère est plus facilement pensée que celle des pères ainsi qu'en atteste les noms choisis pour les institutions « la Protection Maternelle Infantile, les hôtels maternels ». Dans une étude consacrée à la place des pères dans l'action sociale, Nozay souligne qu'aujourd'hui, soit près de 100 ans après leur création, les institutions ont le devoir de s'adapter aux changements de la législation qui ont accompagnéles transformations familiales (2008). Qu'en est-il de cette adaptation qui s'opère nécessairement dans l'après coup, avec un temps de décalage. La sacralisation de la relation mère-enfant dans les accompagnements éducatifs semble avoir limité cette adaptation. On fonctionne sur les stigmates d'une représentation éducative passée. La loi change mais les traditions et les comportements perdurent entérinés, parfois, par des décisions de justice. Pour illustration, nous prendrons le fait que $93 \%$ des décisions de résidence ${ }^{8}$ correspondent à la demande des parents où le plus souvent la résidence est confiée à la mère $(71 \%)$. Donc dans environ trois quarts des cas, les deux parents ont trouvé préférable que ce soit la mère qui détienne la résidence. Ainsi, la préservation du lien mère-enfant prime aux yeux de la mère, du père et de la justice.

$\mathrm{Au}$ vu des résultats obtenus sur la présence des pères dans les institutions, nous pouvons comprendre la complexité à faire évoluer une pensée et des pratiques qui se privent du sujet. En effet, la difficulté des institutions sociales est de penser la place du père en son absence. Cette absence perpétue les représentations de défaillance et de violence $d u$ «père ». Le père présent devient par glissement violence. Et l'institution manque d' « un discernement subtil qui s'im-

8. Rapport du ministère de la justice, publié en novembre 2013, Op. Cit. 
pose pour ne pas laisser s'installer la confusion entre rappeler que le père est lui aussi soumis à la Loi et en profiter pour lui faire la Loi » (Lebrun, op. cit., page 40). Le père présent dans l'institution porte la responsabilité de l'absence de tous les autres. On part du postulat que le père est absent, violent, qu'il faut soutenir la mère dans sa fonction éducative cristallisant ainsi la construction d'un mythe collectif.

\section{Vignette clinique}

Une mesure d'AEMO judiciaire a été prononcée pour un enfant dont les parents sont séparés. La mère a la résidence de l'enfant. Les rythmes de sommeil et l'alimentation de son enfant sont négligés. En effet, une obésité précoce est constatée et il est gêné dans ses apprentissages ainsi que le relève l'école. Le père est violent verbalement envers la mère qu'il juge négligente. Cependant, il est noté qu'il tente d'encadrer le temps de sommeil et l'alimentation ce dont se plaint l'enfant. Il sera décidé que les visites avec le père seront dorénavant médiatisées quand le droit de résidence de la mère est maintenu. Le père a très mal vécu la médiatisation des visites les vivant comme injustes.

La violence du discours est interprétée comme pouvant être le premier pas d'une violence physique. Et ce d'autant plus quand il s'agit du père. La médiatisation des visites qui a vocation à maintenir le lien dans un contexte conflictuel peut dans certains cas conduire à une rupture tant la blessure narcissique peut être grande pour le père et ce quand bien même les intervenants font au mieux pour préserver le lien affectif entre parents-enfants.

Cette contingence scelle le destin déjà périlleux de la place des pères dans les institutions. Si de surcroît la construction fantasmatique vient à être renforcée par l'absentéisme des pères, il devient complexe de modifier cette croyance. L'institution est confortée dans son idée. Et ce d'autant plus qu'elle ne les sollicite pas: pourquoi contacter le père puisqu'il ne fera pas mieux que la mère ?

Interroger cet absentéisme prend son sens. En référence aux travaux de Leprince, nous venons questionner ce en quoi une séparation juridique ${ }^{9}$, 1'intervention d'un service judiciaire, peut venir ébranler le narcissisme des pères. En effet, «les enfants sont (...) le garant de la continuité narcissique » (Leprince, 2010, p. 109). La séparation juridique et physique qu'opèrent les institutions, peut avoir comme corolaire une rupture de cette continuité narcissique et par extension engendrer une dégradation de la transubjectivité (en référence à l'espace transubjectif suscité par l'effet inédit de rencontre entre des subjectivités indépendantes qui luttent contre la perception de la séparabilité de l'autre (Kaës, 1994)). La décision de séparation des services sociaux et juridiques ren-

9. Nous faisons référence aux décisions juridiques suite aux divorces. 
voie au père un sentiment d'illégitimité. Ce qui peut avoir pour effet une dépréciation narcissique. De façon défensive, certains pères répondent sur un mode hétéro-agressif ; aujourd'hui nous observons majoritairement un retrait qui tendrait vers un glissement de «l'oubli du père» (André, Chabert, 2008).

\section{Vignette clinique}

Un signalement avec demande d'AEMO pour préparer un placement concerne une enfant dont la mère est défaillante. Elle en a seule la résidence. L'équipe psycho-sociale assure le suivi de la mesure ordonnée par le magistrat. Un des intervenants, lors d'une réunion, s'interroge : «Le père est-il informé ?». L'éducateur spécialisé se montre étonné : le père n'ayant jamais été présent ni mentionné par la mère il apparaît évident qu'on ne peut compter sur lui. Se pose la question pragmatique de son adresse pour le contacter. «-L'avez-vous demandée à la mère, - Non, mais elle ne voudrait sûrement pas me la donner.» Il s'avère que ce père résidant à l'étranger s'occupe chaque mois d'été de sa fille aux grandes vacances.

Dans cet exemple, on observe qu'un père absent a tendance à être oublié. Or, il y a une obligation juridique d'information et par ailleurs une place symbolique à préserver. Il est considéré que si le père est absent, il sera difficile de le mobiliser. Cela peut être pensé à juste titre mais pour autant il convient d'essayer de le contacter. Il est souvent considéré qu'il est plus blessant pour l'enfant que son père ait été contacté et qu'il n'ait pas répondu plutôt que de ne pas le contacter du tout. Or, un père que l'enfant se représente comme défaillant reste malgré tout une représentation paternelle. La défaillance du père est ressentie par l'enfant et que l'institution en atteste peut lui permettre de mettre du sens sur son ressenti. Cette question reste ouverte et se posera toujours au cas par cas.

\section{Vignette clinique}

Dans le cadre d'une mesure AEMO, une semaine en colonie de vacances est proposée à l'enfant pendant le temps de résidence de la mère. Les parents séparés exercent un droit de résidence alternée. Le père a la garde de l'enfant un week-end sur deux et la moitié des vacances scolaires. La colonie ayant été proposée sur la semaine où l'enfant est avec sa mère, le père ne sera pourtant pas prévenu dans un premier temps. Le père ayant mis à mal les précédentes propositions de l'institution, il a été jugé préférable de ne pas l'avertir dès le début.

Rappelons qu'un droit de résidence exercé sur la majorité du temps par un parent ne veut pas dire qu'il s'agit d'un droit de garde exclusif mais réparti. Un père moins présent parce qu'il a moins la garde de l'enfant n'est pas pour autant absent. Il semble parfois s'opérer un glissement de ce type. Mais l'intérêt de l'enfant primant, il peut être nécessaire dans certaines situations de s'adapter. 
Les vignettes cliniques décrites brièvement ont été choisies pour leur caractère paradigmatique sans la vaine prétention qu'elles résument à elles seules les multiples enjeux de la place des pères dans les institutions, aussi nombreux qu'il $y$ a de pères etd'enfants.

\section{CONCLUSION}

Il semble que les représentations des pères dans les institutions soient trop souvent cantonnées à des positions abandonniques ou violentes. La construction de ce mythe trouve ses sources dans le fondement même des institutions. Si les lois, les modèles familiaux et les responsabilités parentales ont évolué, les représentations archaïques de la fonction paternelle se sont enkystées. Dans une société où une égalité démocratique des responsabilités parentales s'est installée, il est clair qu'elle fait figure de réel dans les situations que nous qualifierons de non problématiques. En cas de conflit, le maintien de l'équilibre du couple parental devient un travail périlleux et la fonction parentale se voit déchirée. Le mythe du couple parental parfait suppose une mère suffisamment bonne répondant à ses fonctions affectives, éducatives et un père cadrant, autoritaire. Ajoutons que cette étude ne tient pas compte de l'âge des enfants concernés et que, dans des travaux ultérieurs, il faudra sans doute tenir compte des fonctions différentes du père, selon qu'il s'agit du père pré-œdipien (contextualisateur et nourricier de la dyade) ou du père post-œdipien (castrateur et séparateur).

Nous constatons une lente évolution des représentations du père dans les institutions. La pérennisation des représentations est, nous semble-il, étroitement liée à la moindre présence des pères, auprès de leurs enfants et au sein des institutions. Mais cette désertification ne serait-elle pas l'expression d'une faille narcissique en lien avec leur nouvelle position déchue de père ? Ainsi, le travail institutionnel ne devrait-il pas s'orienter vers une structuration d'un modèle d'accompagnementet de soutien aux failles narcissiques générées parles conflits conjugaux et qui trouvent à s'exprimer dans leurs fonctions parentales ? Ceci permettrait un rééquilibrage des fonctions éducatives en accompagnant le père. Si le soutien des pères dans leurs fonctions parentales semble être une nécessité première, il doit s'appuyer sur les références que la société se fait de cette place. Aujourd'hui, la place des pères est complexe car le modèle qui les représentait s'est délité avec le temps sur le plan juridique mais pas sur le plan fantasmatique. Face à cette absence de modèle, ce n'est pas le père seul qui est en difficulté, l'institution l'est également, car elle se réfère à un idéal dépassé pour penser sa place dans le présent. Aujourd'hui, le père doit créer sa place au sein de la famille. Alors que les mères évoluent plus dans la continuité du modèle archaïque de leur fonction maternante au sein de la famille. 
L'enjeu est donc de reparentaliser les pères en ne les limitant pas à un rôle de pourvoyeur économique souvent mis à mal dans les situations rencontrées en AEMO.

La loi change, les mentalités évoluent lentement fixées à certains éléments du passé, et les institutions sont aux prises avec ces évolutions aux temporalités différentes.

\author{
Aziz ESSADEK \\ azizessadek@gmail.com \\ Adèle ASSOUS \\ adeleassous@hotmail.fr \\ Bernard GOLSE \\ bernard.golse@aphp.fr \\ Magali CHABIN-FENLON \\ sse91@olgaspitzer.asso.fr \\ Corinne FERNET-LUCAS \\ sse91@ olgaspitzer.asso.fr
}

\title{
BIBLIOGRAPHIE
}

ANDRÉ, J., Chabert C. (2004), L'oubli du père, Paris, PUF.

Clément, R., (1986), «La séparation : une réponse aux phénomènes de dysparentalité, »,

Les communications médicales: Actes du congrès afirem (Angers), 164-165.

Corbin, A., Courtine, J.-J., Vigarello, G. (2011), Histoire de la virilité, Paris, Seuil.

Delion, P., (2011), La fonction parentale, Bruxelles, Fabert.

Dor. J., (1998), Introduction à la lecture de Lacan, Paris, Denoël.

FÉdidA, P., VILLA, F., (1999), Le cas en controverse. Monographies de psychopathologie, Paris, PUF.

Ferrand, M., (2004), Féminin, masculin, Paris, La Découverte, Collection Repères.

Ferrand, M., (2005), «Égaux face à la Parentalité ? Les résistances des hommes... et les réticences des femmes », Actuel Marx, 37, 71-88.

Freud, S., (1909), Le roman familial des névrosés, Névrose, psychose et perversion, Paris, PUF, $5^{\circ}$ édition 1985, 157-160.

Golse, B., (1990), Insister, exister. De l'être à la personne, Paris, PUF.

HérItIER, F., (2012), Masculin/Féminin II, Dissoudre la hiérarchie, Paris, Odile Jacob.

JACOB AlBy, V., Vives, J.-M., (2015), « Parentalité et paternité : les nouvelles modalités contemporaines du «faire-famille »», Dialogue, 207, 19-30.

Kaes, R., (1994), La parole et le lien. Processus associatifs et travail psychique dans les 
groupes, Dunod Paris, $2^{\mathrm{c}}$ édition, 2005.

Lebrun, J.-P. (2011), Fonction Maternelle Fonction Paternelle, Bruxelles, Fabert. LEPRINCE, C., (2010, «Le divorce: ses conséquences psychiques dans les liens familiaux », in Le divan familial, 24, 109-122.

NozAy, C., (2008), « La place du père dans l'action sociale : l'exemple des centres maternels », Enfances \& Psy, 41, 140-147.

Senécal, I., Garon, R., SAucier, J.-F., (2015), « Le travail sur les représentations des lignées paternelle et maternelle lors de l'accès à la paternité. Recherche exploratoire, La psychiatrie de l'enfant, Vol. 58, nº2, p. 453-504.

Vicente, C., Schom, A.-C., Robert, P., (2014), «L'institution et la place des familles en protection de l'enfance », Dialogue, 204, 35-46.

\title{
Aziz Essadek, Adèle Assous, Bernard Golse, Magali Chabin-Fenlon, Corinne Fernet- Lucas - Entre idéal dépassé et norme imposée: une représentation figée du père proje- tée dans une société en mutation. Quelle représentation de la fonction paternelle dans une institution sociale exerçant des mesures d'AEMO?
}

Résumé : L'évolution des lois sur l'autorité paternelle puis parentale a généré une modification des fonctions du père. Mais qu'en est-il des représentations : ont-elles évolué dans la même temporalité ? Nous interrogerons les effets du passage d'une autorité strictement paternelle à parentale. Construire un nouveau modèle paternel semble être un projet périlleux, parfois inconfortable, pour les pères eux-mêmes, les mères mais également les institutions.

L'article propose de montrer, à partir d'une historisation de l'évolution juridique de la place des pères dans la société et d'une étude populationnelle réalisée auprès de 796 mineurs au sein d'un service mettant en œuvre des mesures d'AEMO (Assistance Éducative en Milieu Ouvert), que la place des pères dans les institutions sociales est pensée à partir d'une représentation passée, ce qui impacte l'accompagnement et le travail de reparentalisation des pères.

Mots-clés : Autorité - Parentalité - Père - Blessure narcissique - Oubli du père.

\begin{abstract}
Aziz Essadek, Adèle Assous, Bernard Golse, Magali Chabin-Fenlon, Corinne FernetLucas - Between an Outdated Ideal and Imposed Norms: A Solidified Representation of Fatherhood in a Changing Society. What representation of fatherhood in a social institution such as the EAOE?
\end{abstract}

Abstract : The evolution of laws on paternal and parental authority has generated modifications in the father's functions. But what about paternal representations - have they evolved at the same time? We shall examine the move from purely paternal to parental authority. Building a new paternal model is a perilous, even uncomfortable project for fathers themselves, but also for mothers and institutions.

This article adopts the perspective of a historical approach to legal changes effecting fatherhood in society, as part of a population study carried out with 796 minors from an 
EAOE service (Educational Assistance in an Open Environment). This approach demonstrates that the representation underpinning the role of fathers in social institutions is outdated. This makes supporting and accompanying them a complex task, and impacts on the process of returning fathers to their roles as parents.

Key-words : Authority - Parenthood - Father - Narcissistic wound - Forgetting the father 